DOI: https://doi.org/10.33103/uot.ijccce.20.2.3

\title{
Classification of Multi Heart Diseases With Android Based Monitoring System
}

\author{
Alaa Al-Obaidi ${ }^{1}$, Satea .H Alnajjar ${ }^{2}$ Mohamed Nsai ${ }^{3}$, Hassan Sharabaty ${ }^{4}$ \\ ${ }^{1,2,3}$ College of Engineering, AL-Iraqia University, Baghdad, Iraq \\ ${ }^{4}$ Dept. of Electrical \& Electronics Eng., University of Turkish Aeronautical Association, Ankara, Turkey \\ alaa.alobaide@aliraqia.edu.iq, sharabaty@ieee.org
}

\begin{abstract}
Electrocardiogram (ECG) examination via computer techniques that involve feature extraction, pre-processing and post-processing was implemented due to its significant advantages. Extracting ECG signal standard features that requires high processing operation level was the main focusing point for many studies. In this paper, up to 6 different ECG signal classes are accurately predicted in the absence of ECG feature extraction. The corner stone of the proposed technique in this paper is the Linear predictive coding (LPC) technique that regress and normalize the signal during the pre-processing phase. Prior to the feature extraction using Wavelet energy (WE), a direct Wavelet transform (DWT) is implemented that converted ECG signal to frequency domain. In addition, the dataset was divided into two parts, one for training and the other for testing purposes Which have been classified in this proposed algorithm using support vector machine (SVM). Moreover, using MIT AI2 Companion was developed by MIT Center for Mobile Learning, the classification result was shared to the patient mobile phone that can call the ambulance and send the location in case of serious emergency. Finally, the confusion matrix values are used to measure the proposed classification performance. For 6 different ECG classes, an accuracy ration of about $98.15 \%$ was recorded. This ratio became $100 \%$ for 3 ECG signal classes and decreases to $97.95 \%$ by increasing ECG signal to 7 classes.
\end{abstract}

Index Terms-Abnormal ECG; Discrete Wavelet Transform; Linear Prediction Coding; Multi Heart Diseases classification; Support Vector Machines.

\section{INTRODUCTION}

A heart diagnosis disease was considered as the major issue in clinical and biological systems [1]. Post-processing, pre-processing and feature extraction procedures were implemented for Electrocardiogram (ECG) examination [2]. ECGs has been classified by many researchers into two classes normal and abnormal related to heart cases [3]. A comparison study between the extracting of ECG features and the standard ECG normal features in terms of ST segment, QRS complex, $\mathrm{P}$ wave, $\mathrm{T}$ wave and $\mathrm{PR}$ interval was presented by many works [4][5]. In this comparison study, the classified classes were increased and in the same time, some diseases in abnormal ECG were recognized. However, in some diseases that required high level processing operation such as Ventricular fibrillation, extracting standard features exhibited high complexity [6]. On the other side, many studies avoided the complexity of extracting the standard features of ECG related to heart diseases classification [7][8]. Using such techniques, by increasing the number of the classes, the accuracy was decreased, in the same time, the degradation of the accuracy was proportionally to the increment of the classes number up to 6 heart diseases high accuracy 
classes is presented in this study. Linear predictive coding (LPC) is proposed in order normalize and avoid signal redundancy in ECG signal during analyzing and preprocessing phase. Discrete Wavelet transform (DWT) is used for transferal of ECG signal into the frequency domain with 8 different levels. In addition, the energy features for these 8 levels of the analyzed ECG signal is extracted using Wavelet energy (WE). Moreover, in terms of classification phase, the test samples are classified, and datasets is trained using support vector machine (SVM). Finally, a confusion matrix equation is used to evaluate the proposed algorithm performance.

\section{METHODOLOGY}

\section{A. Linear Predictive Coding (LPC)}

The first step of the proposed algorithm, which corresponds the preprocessing phase, is dedicated to prepare the ECG samples by removing the noise and standardize the variable values and samples period by using Linear Prediction Coding with standardization of the signal value to be laid between -1 and +1 .

LPC stands for customary mathematical functions in which forthcoming magnitudes of the digital signal are predicted as a linear combination of past values without a pre-selected kernel. The main advantage of not using a kernel is that you are not biasing the shape of your signal to be similar to an arbitrary, pre-selected shape. Linear Predictive Coding is disjointed into dual parts of linear predictive coefficients and the prediction error. The predictor function equation can be given asError! Reference source not found.[9]:

$$
s[n]=\sum_{k=1}^{p} a_{k} s[n-k]
$$

Where $s[n]$ stands for predicated value in need, while $a_{k}$ represents the coefficient predicator. For LPC, three steps are required including autocorrelation, reflection coefficient, and Levinson Durbin algorithms.

Below will discuss each step in details math model

\section{1) Autocorrelation Coefficient}

The conception of the autocorrelation coefficient has been used for measuring the sum of linear correlations among the remarks of dual data intervals. The autocorrelation coefficient takes numeric magnitudes that are positioned amid +1 and -1 . The magnitude of +1 stands for the observed data possess a strong trend in the positive inclination, while the magnitude of -1 stands for the observed data possess a strong inclination in the negative trend [10]. The correlation amid any dual values of several arbitrary sequence such $1 \ldots \mathrm{N}-1$, has measured as an autocorrelation coefficient for this sequence.

If autocorrelation coefficient of sequence is of time series, it will be changed in sequence, where the calculated correlation amid single time series and next one of time units. First-order autocorrelation coefficient stands for uncomplicated correlation coefficient of $1^{\text {st }}$ unit that is $x t, t=1,2, \ldots, N-1$ and the next unit is $x t, t=2,3, \ldots, N$, then the correlation amid $x t$ and $x t+1$ is specified through:[11]

$$
r_{1}=\frac{\sum_{t=1}^{N-1}\left(x_{t}-\bar{x}_{(1)}\right)\left(x_{t+1}-x_{(2)}\right)}{\left[\sum_{t=1}^{N-1}\left(x_{t}-\bar{x}_{(1)}\right)^{2}\right]^{\frac{1}{2}}\left[\sum_{t=2}^{N}\left(x_{t}-\bar{x}_{(2)}\right)^{2}\right]^{\frac{1}{2}}}
$$

Where $\overline{\mathrm{x}}_{(1)}$ stands for the $1^{\text {st }}$ magnitude of $\mathrm{N}-1$ and $\overline{\mathrm{x}}_{(2)}$ is the last unit of $\mathrm{N}-1$, 


\section{2) Reflection Coefficient}

The $2^{\text {nd }}$ step in LPC algorithm is to discover a reflection coefficient of resultant autocorrelation coefficient. The elementary description of reflection coefficient is the amplitude variance of the reflected wave divided by the sum of $\mathrm{v}$ amplitude of the happening wave based on Eqs. (3) and (4) [12].

$$
\begin{aligned}
& R . c=\frac{\text { amplitude of the reflected wave }}{\text { amplitude of the incident wave }} \\
& \qquad \mathrm{Rc}=\frac{\mathrm{V}_{2}-\mathrm{V}_{1}}{\mathrm{~V}_{2}+\mathrm{V}_{1}}
\end{aligned}
$$

\section{3) Levinson Durbin Algorithm}

Levinson Durbin Algorithm (LDA) is employed for solving identical dimension of linear matrix, that requires several predictor orders and the recursion is still processed till reaching the latest predictor Error! Reference source not found.[12].

$$
\begin{aligned}
k_{i} & =\frac{\left(E(i)-\sum_{j=1}^{i-1} \alpha_{j}^{i-1} E(i-j)\right.}{E^{i-1}} \\
E^{i} & =\left(1-k_{i}^{2}\right) E^{i-1}
\end{aligned}
$$

Where E stands for the predicted error, and $\alpha$ stands for prediction coefficient.

\section{B. Feature Extraction by DWT along with Wavelet Energy}

The second phase of the proposed algorithm is dedicated to extract the signal features by decomposing the LPC output into 8 levels using Discrete Wavelet Transform (DWT) in order to reduce the samples dimension which give better classification results in the following phases. Wavelet transform is an effective time-frequency analysis tool. To implement DWT, two principal methods exist, Filter Banks method based on the frequency domain and Lifting Scheme (LS) method based on the time domain [13].

\section{1) Filter Bank Method (FB)}

FB technique, in solitary level of discrete wavelet decomposition, where the input signal was divided into dual amounts of frequency which pass these parts at the equal time in a pair of low pass $\mathrm{H}(\mathrm{z})$, and high pass $\mathrm{G}(\mathrm{z})$, filters. The FB method realizes the DWT by convolving filter with taps and samples of the input signal. Both $\mathrm{H}(\mathrm{z})$ and $\mathrm{G}(\mathrm{z})$ filters are designated by Eqs. (7) and (8) [13]:

$$
\begin{aligned}
& \mathrm{H}(\mathrm{z})=\mathrm{h}_{0}+\mathrm{h}_{1^{\mathrm{z}^{-1}}}+\mathrm{h}_{2^{\mathrm{z}^{-2}}}+\cdots+\mathrm{h}_{\mathrm{N}^{\mathrm{z}^{-N}}} \\
& \mathrm{G}(\mathrm{z})=\mathrm{g}_{0}+\mathrm{g}_{1^{\mathrm{z}^{-1}}}+\mathrm{g}_{\mathrm{2}^{\mathrm{z}^{-2}}}+\cdots+\mathrm{g}_{\mathrm{M}^{\mathrm{z}^{-M}}}
\end{aligned}
$$

\section{2) Lifting Structure (LS) Method}

As shown in Figure 1, LS method stands for execution wavelets based on the time domain. The LS structure divides signal sample into even and odd samples. At that point, $\mathrm{P}$ stands for a function that used in even samples as a prediction function to predict the odd samples from even samples. The $P$ function generates the details coefficient (d). The $U$ is a function applied on detail signal and gathering the result of details coefficient (d) with an even samples and the output is approximation coefficients (s).The equations of $d$ and s function are defined in below [9].

$$
\mathrm{d}=\mathrm{X}_{\mathrm{odd}}-\mathrm{P}\left(\mathrm{X}_{\text {even }}\right)
$$




$$
\mathrm{s}=\mathrm{X}_{\text {even }}+\mathrm{U}(\mathrm{d})
$$

where: $\mathrm{x}=$ input signal

$\mathrm{s}=$ approximation coefficient of $\mathrm{x}$

The $d$ and $s$ coefficients in lifting scheme, correspondingly, are the similar as high pass and low pass outputs in FB [13].

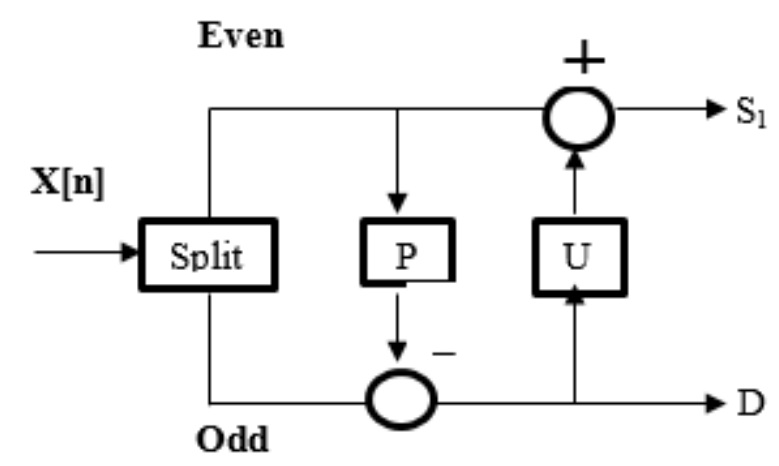

Figure 1: The Method of Lifting SCHEME

Figure 2 shows how DWT is used to decompose the LPC output into 8 levels. The output of each level is group of dual coefficients, namely approximation (cA) and details (cD). At the end of the decomposition level, the features vector or the DWT coefficients vector is created from the approximation coefficient of the last level i.e. cA8 and from the details coefficients of all level.

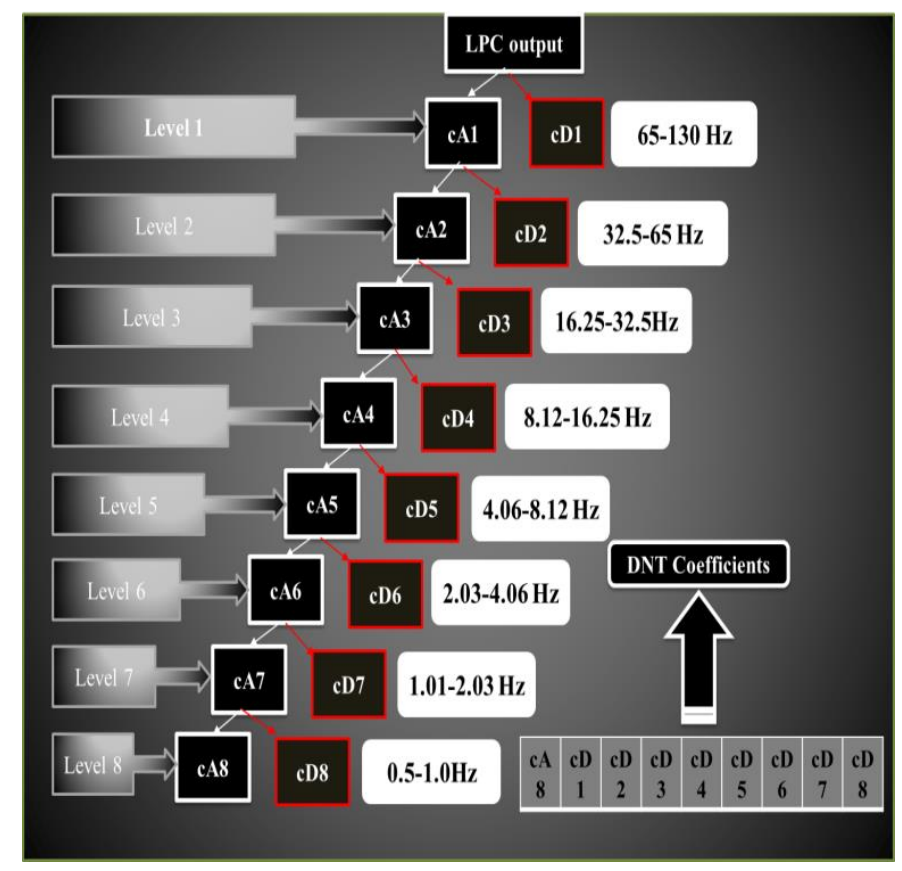

FiguRE 2: DECOMPOSITION THE LPC OUTPUT INTO 8 LEVELS VIA DWT

\section{3) Wavelet Energy}

In the previous section, DWT generates number of feature vectors for each level by find the approximation and details coefficients. The total length of feature vector still large as length of input data ECG signal, To find the energy of each resulted coefficient gives less vectors as defined in equation (11) [14].

$$
\mathrm{E}_{\mathrm{m}}=\sum_{\mathrm{n}} \operatorname{coef}_{\mathrm{n}}^{2}
$$


Where $\mathrm{m}$ stands for the amount of levels, $\mathrm{n}$ is total number of coefficients (approximation and details).

\section{Support Vector Machine (SVM)}

SVM stands for an accurate learning tool functional in data classification with high efficiency. The method of SVM is to determine the extreme margin for dual classes of data [15].

The basic principle of SVM is summarized by dividing the dataset samples into 2 groups: Training samples and test samples. The input data of the training part is s parted into dual classes in $2 \mathrm{D}$ plane in order to find the optimal separation line between them [16]

$$
X=\left\{x_{1 i} y_{1 i}, x_{2 j} y_{2 j}\right\}
$$

where: $i=1, \ldots, N_{1}$

$$
j=1, \ldots, N_{2}
$$

$X=$ the datasets having dual classes

$x_{1 i}$ determines the $1^{\text {st }}$ class

$x_{2 j}$ determines the $2^{\text {nd }}$ class

$y_{l i}$ defines the labels for $1^{\text {st }}$ class with labels 1

$y_{2 j}$ defines the labels for $2^{\text {nd }}$ class with labels 2

The result $\mathrm{y}(\mathrm{x})$ for each class label is defined as [17]:

$$
\begin{cases}w^{T} x+b \geq+1, & \text { if } y(x)=+1 \\ w^{T} x+b \leq-1, & \text { if } y(x)=-1\end{cases}
$$

The last step of classification is to find the closest training sample that best matches the test one. So, the classifier will return the most matching class name (label). The classification steps using SVM are shown in Figure 3.

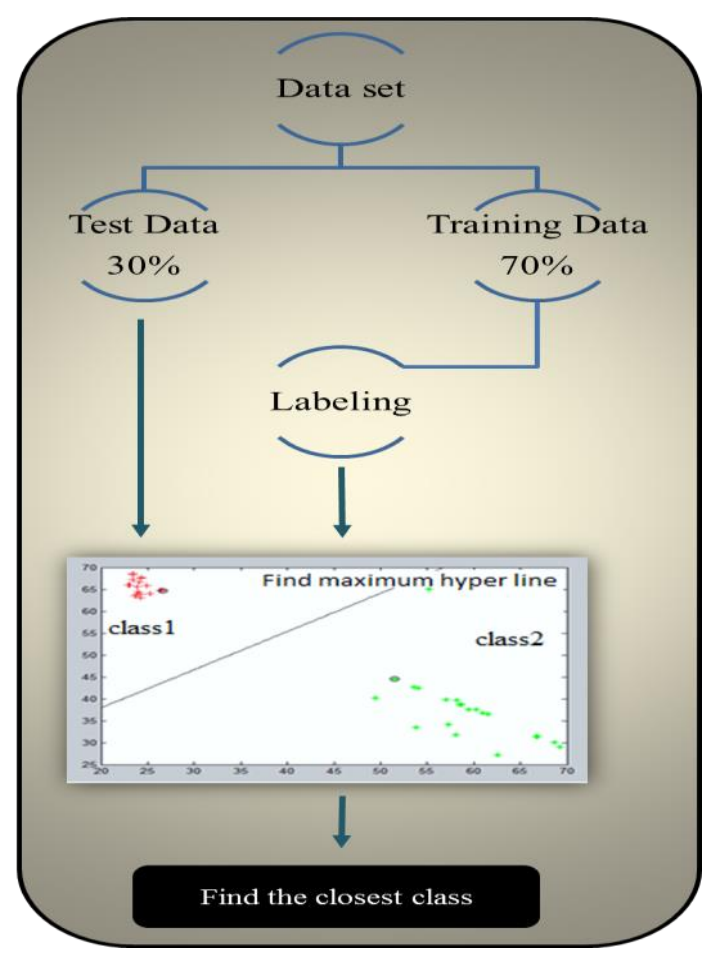

FiguRE 3: CLASSIFICATION USING SVM 


\section{The Quality Performance Measurement}

For determining the performance of the projected algorithm, confusion matrix method is employed to show the representation of both actual and predicted classes as seen in Table 1, where TP stands for True Positive prediction and represents the number of positive classified cases, which are found positive in the real dataset. TN stands for True Negative classification and corresponds the correct prediction number of the negative data. On the other hand, FP stands for False Positive and represents the number of wrong prediction cases of positive data. FN, or False Negative, gives the amount of patterns classified as negative whereas they are positive in the reality [18].

TABLe 1.COnfusion Matrix

\begin{tabular}{|c|c|c|}
\hline & Positive predicted & Negative predicted \\
\hline Positive actual & $\mathrm{TP}$ & FN \\
\hline Negative ctual & FP & TN \\
\hline
\end{tabular}

The performance parameters, namely Sensitivity, Specificity, Accuracy, and Precision can be evaluated by means of confusion matrix values [18]:

$$
\begin{aligned}
& \text { sensitivity }=\frac{T P}{T P+F N} \\
& \text { accuracy }=\frac{T P+T N}{T P+T N+F P+F N} \\
& \text { specificity }=\frac{T N}{F P+T N} \\
& \text { precision }=\frac{T P}{T P+F P}
\end{aligned}
$$

\section{E. Android based Remote Monitoring System}

After delivering the classification result, we use MIT App Inventor developed by MIT Education Center to share our diagnostic of the heart disease with any Android based operating system like the patient mobile phone. The transfer process is achieved through MIT server (ai2.appinventor.mit.edu) by using AI2 Companion developed by MIT Center for Mobile Learning and available in Google Play Store. The main goal of the proposed mobile monitoring system is for alerting the nearby healthcare center in case of life-threatening heart condition detected or call directly the ambulance and send the patient's location. Figure 4 show a screenshot to the mobile phone after receiving the diagnostic result from the computer.

\section{RESULTS AND DISCUSSIONS}

The analyzed dataset in this paper is collected from PhysioNet.org, which is considered the biggest research resource for complex physiologic signals [19]. The collected ECG records comprise of 7 ECG classes whose length equals $8400 \mathrm{sec}$. Each class, of the selected database, represents different heart disease with a total number of 120 samples, i.e. 98 samples for training the classifier and 42 samples to test the classification results. 


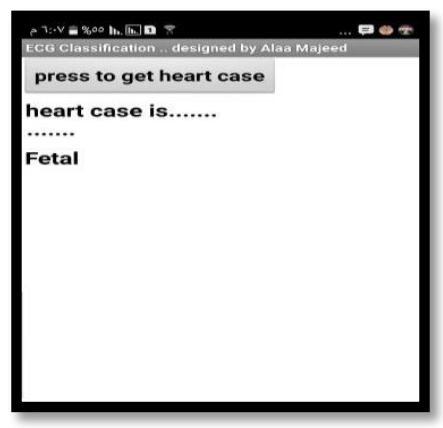

FigURE 4: SCREENSHOT OF RECEIVING THE DIAGNOSTIC RESULT.

To evaluate the classification performance of the proposed algorithm, the confusion matrix values were measured in both cases, with and without LPC carrying out in the preprocessing stage. Table 2 shows the performance parameters of classifying 7 different classes without applying LPC. Whereas Table 3 presents the performance of the same classes when implementing LPC in the preprocessing phase. It is clear that the overall average accuracy increased from $92.59 \%$ to $97.95 \%$ after applying LPC. The low classification accuracy of class 5 and class 7 can be explained by the similarity of ECG signals of these classes because both of them are related to fetal phonocardiograph.

TABLE 2.CLASSIFICATION RESULTS FOR 7 DIFFERENT CLASSES WITHOUT LPC

\begin{tabular}{cccc}
\hline & Specificity $\%$ & Sensitivity $\%$ & Accuracy \% \\
\hline Class 1 & 100 & 35.3 & 73.8 \\
Class 2 & 100 & 75 & 95.23 \\
Class 3 & 97.22 & 83.33 & 95.23 \\
Class 4 & 94.6 & 80 & 92.85 \\
Class 5 & 85.71 & 100 & 85.71 \\
Class 6 & 97.22 & 83.33 & 95.23 \\
Class 7 & 85.71 & 100 & 85.71 \\
OVERALL & $\mathbf{9 3 . 6 5}$ & $\mathbf{6 1 . 9}$ & $\mathbf{8 9 . 1 1}$ \\
AVERAGE & & & \\
\hline
\end{tabular}

TABLE 3.CLASSIFICATION RESULTS FOR 7 DIFFERENT CLASSES USING LPC

\begin{tabular}{cccc}
\hline & Specificity $\%$ & $\begin{array}{c}\text { Sensitivity } \\
\text { \% }\end{array}$ & Accuracy \% \\
\hline Class 1 & $100 \%$ & $85.7 \%$ & $97.62 \%$ \\
Class 2 & $100 \%$ & $85.7 \%$ & $97.62 \%$ \\
Class 3 & $100 \%$ & $85.7 \%$ & $97.62 \%$ \\
Class 4 & $94.73 \%$ & $100 \%$ & $95.2 \%$ \\
Class 5 & $100 \%$ & $100 \%$ & $100 \%$ \\
Class 6 & $100 \%$ & $100 \%$ & $100 \%$ \\
Class 7 & $97.3 \%$ & $100 \%$ & $97.61 \%$ \\
& & & \\
OVERALL & $\mathbf{9 8 . 8 \%}$ & $\mathbf{9 2 . 8 5 \%}$ & $\mathbf{9 7 . 9 5 \%}$ \\
AVERAGE & & & \\
\hline
\end{tabular}


It should be mentioned here that decreasing the number of classes will increase the overall classification accuracy up to $100 \%$ even in case of classifying similar classes namely, class 5 and class 7. Table 4 compares between the achieved accuracy when classifying 3 classes with and without using LPC.

TABLE 4.ClASSIFICATION RESULTS FOR 3 DIFFERENT CLASSES WITH AND WITHOUT USING LPC

\begin{tabular}{ccc} 
& Accuracy - without LPC & Accuracy - with LPC \\
\hline Class 1 & $100 \%$ & $100 \%$ \\
Class 3 & $83.33 \%$ & $100 \%$ \\
Class 5 & $83.33 \%$ & $100 \%$ \\
OVERALL & $\mathbf{9 0 . 4 8 \%}$ & $\mathbf{1 0 0 \%}$ \\
AVERAGE & & \\
\hline
\end{tabular}

TABLE 5.COMPARISON WITH THE PREVIOUS STUDIES

\begin{tabular}{lccc}
\hline \multicolumn{1}{c}{ Author } & $\begin{array}{c}\text { Classes } \\
\text { number }\end{array}$ & Accuracy & Year \\
\hline Melgani [20] & 5 & $85.98 \%$ & 2008 \\
Korurek[21] & 6 & 96.3 & 2010 \\
Das [22] & 5 & $97.5 \%$ & 2014 \\
Desai [23] & 5 & 98.4 & 2016 \\
Ahmad [8] & 5 & $97.9 \%$ & 2018 \\
Yinsheng Ji[5] & 5 & $99.21 \%$ & 2019 \\
Thiswork & $3,6,7$ & $100 \%, 98.15 \%, 97.95 \%$ & 2019
\end{tabular}

\section{CONCLUSION}

In this study, ECG classes have been normalized and regressed into the same amplitude within preprocessing phase using LPC algorithm. Up to 7 different heart diseases classes have been highly distinguished and submitted the diagnosing result to Android based system that have the ability of calling the nearest healthcare center and send the patient's location.

All the dataset was delivered using Physionet.org and implemented via MATLAB software package. Regarding to ECG feature extraction, at first, DWT has converted the analyzed signal into 8 levels. In addition, the classification of ECG classes was achieved using SVM. Moreover, in case of with and without LPC, the confusion matrix was used for all evaluated classes. Related to the obtained results, the proposed method in this study, highly overall average accuracy was achieved in case of 6 and 7 ECG glasses and up to $98.15 \%$ and $97.95 \%$ was obtained respectively. Finally, the total resolution of $100 \%$ was achieved for 3 different classes.

\section{REFERENCES}

[1] S. Jokic, I. Jokic, S. Krco, and V. Delic, "ECG for Everybody: Mobile Based Telemedical Healthcare System," in ICT Innovations 2015, Advances in Intelligent Systems and Computing, vol. 399, S. Loshkovska, and S. Koceski, Ed. Cham: Springer, 2016, pp. 89-98.

[2] S. Karpagachelvi, M. Arthanari, and M. Sivakumar, "ECG Feature Extraction Techniques - A Survey Approach," International Journal of Computer Science and Information Security, vol. 8, pp. 76-80, April 2010.

[3] C. I. M. Cruz, J. P. Marasigan, A. P. G. Perez, J. E. V. Pillejera, N. P. Veron and A. R. dela Cruz, "A comparative study between DWT-ANFIS and DWT-SVM in ECG classification," in 2016 IEEE Region 10 Conference (TENCON), Singapore, 2016, pp. 2052-2055. 
[4] S. Subbiah, R. Patro, and P Subbuthai, "Feature Extraction and Classification for ECG Signal Processing based on Artificial Neural Network and Machine Learning Approach," in International Conference on Inter Disciplinary Research in Engineering and Technology, 2015, pp. 50-57.

[5] Yinsheng Ji , Sen Zhang and Wendong Xiao ," Electrocardiogram Classification Based on Faster Regions with Convolutional Neural Network", Sensors 2019, 19, 2558.

[6] A. H. Ismail, M. Fries, R. Rossaint, and S. Leonhardt, "Validating the Reliability of Five Ventricular Fibrillation Detecting Algorithms," in 4th European Conference of the International Federation for Medical and Biological Engineering. IFMBE Proceedings, vol. 22, J. Vander Sloten, P. Verdonck, M. Nyssen, J. Haueisen, Ed., Berlin: Springer, 2009.

[7] F. Melgani and Y. Bazi, "Classification of Electrocardiogram Signals with Support Vector Machines and Particle Swarm Optimization," in IEEE Transactions on Information Technology in Biomedicine, vol. 12, no. 5, pp. 667-677, Sept. 2008.

[8] A. S. Ahmad ,M. S. Matti, A. S. Essa, O. A. ALhabib, S. Shaikhow, " Features Optimization for ECG Signals Classification", (IJACSA) International Journal of Advanced Computer Science and Applications, Vol. 9, No. 11, 2018, $\mathrm{P}$ a g e 383 .

[9] M. M. Azmy Gad, "Classification of mental tasks using support vector machine based on linear predictive coding and new mother wavelet transform," in 2015 International Conference on Biomedical Engineering and Computational Technologies (SIBIRCON), Novosibirsk, 2015, pp. 156-159.

[10] J. W. Gooch, Encyclopedic Dictionary of Polymers. Springer Science LLC, 2011.

[11] C. Chatfield, The Analysis of Time Series: An Introduction, Sixth Edition.Chapman and Hall CRC, 2016.

[12] L. R. Rabiner, and R. W. Schafer, "Introduction to Digital Speech Processing", Foundations and Trends in Signal Processing, Vol. 1, Issue 1, January 2007.

[13] S. A. Salehi and R. Amirfattahi, "VLSI Architectures of Lifting-Based Discrete Wavelet Transform," in Discrete Wavelet Transforms - Algorithms and Applications, H. Olkkonen, Ed., InTech, 2011, pp. 41-56.

[14] G. Garg, V. Singh , J.R.P Gupta and A.P. Mittal, " Relative Wavelet Energy As A New Feature Extractor For Sleep Classification Using Eeg Signals", International Journal of Biomedical Signal Processing, 2(2), 2011, pp. 75-80.

[15] S. Abe, Support Vector Machines for Pattern Classification, Springer Press, 2010.

[16] N. Mehra and S. Gupta, "Maximal margin multi-classifier based on SVM hyperparameter tuning," in 2015 International Conference on Computer, Communication and Control (IC4), Indore, 2015, pp. 1-5.

[17] J. A. K. Suykens, and J. V. Gestel, Least Squares Support Vector Machines, Singapore: World Scientific, 2005.

[18] D. L. Olson, D. Delen, Advanced Data Mining Techniques, Springer, 2008.

[19] A. L. Goldberger, L. A. N. Amaral, L. Glass, J. M. Hausdorff, P. Ch. Ivanov, R. G. Mark, J. E. Mietus, G. B. Moody, C. K. Peng, H. E. Stanley, "PhysioBank, PhysioToolkit, and PhysioNet: Components of a New Research Resource for Complex Physiologic Signals,” Circulation, vol. 101(23), pp. 215-220, June 2000.

[20] F. Melgani and Y. Bazi, "Classification of Electrocardiogram Signals with Support Vector Machines and Particle Swarm Optimization, " in IEEE Transactions on Information Technology in Biomedicine, vol. 12, no. 5, pp. 667-677, Sept. 2008.

[21] M. Korürek and B. Doğan, "ECG beat classification using particle swarm optimization and radial basis function neural network," Expert Systems with Applications, vol. 37, no. 12, pp. 7563-7569, 2010.

[22] M. K. Das and S. Ari, "ECG Beats Classification Using Mixture of Features," International Scholarly Research Notices, vol. 2014, Article ID 178436, 12 pages, 2014.

[23] U. Desai, R. J. Martis, C. G. Nayak, Sarika K. and G. Seshikala, "Machine intelligent diagnosis of ECG for arrhythmia classification using DWT, ICA and SVM techniques," in 2015 Annual IEEE India Conference (INDICON), New Delhi, pp. 1-4, 2015. 